\title{
Effective field theory approach to pionium
}

\author{
D. Eiras* and J. Soto \\ Departament d'Estructura i Constituents de la Matèria and Institut de Física d'Altes Energies, Universitat de Barcelona, Diagonal, \\ 647 E-08028 Barcelona, Catalonia, Spain
}

(Received 11 June 1999; published 11 May 2000)

\begin{abstract}
The various dynamical scales below the pion mass involved in $\pi^{+} \pi^{-}$atoms are sequentially integrated out using nonrelativistic effective field theory techniques. This allows us to systematically organize the corrections to the energy levels and decay width. We present our results in terms of a single unknown constant which may be obtained by matching to the chiral Lagrangian with electromagnetic interactions at two loops.
\end{abstract}

PACS number(s): 12.39.Fe, 11.10.St, 13.75.Lb, 36.10.Gv

\section{INTRODUCTION}

Hadronic atoms have attracted much interest for a long time [1]. Typically there is an interesting interplay between strong and electromagnetic interactions. Whereas the latter are responsible for the bound state formation, the former produce their decay. Although the treatment of electromagnetic interactions is based on solid theoretical grounds, this is not so for the strong interactions. Traditionally, the latter are modeled by various types of short range potentials [2]. Although this is usually enough to fit the available data, it would be desirable to have a more direct connection with what is believed to be the fundamental theory of strong interactions, namely QCD. This is becoming even more urgent since the current DIRAC experiment at CERN [3], which plans to measure the pionium decay width at $10 \%$ accuracy [4], is meant to extract the pure hadronic pion-pion scattering lengths, which may, in principle, be obtained from QCD.

It has become apparent during the past decade that the most fruitful way to approach low energy strong interaction physics from QCD is not by direct calculations from this theory but going through intermediate effective field theories (EFT), which are equivalent to QCD in a particular range of energies. For instance the chiral Lagrangian $(\chi L)[5]$ is an EFT for pions, which is equivalent to QCD for energies below the rho mass. The EFTs typically depend on various unknown constants, which in principle may be obtained from the fundamental theory. In practice, this may sometimes be achieved, such as for instance in the case of nonrelativistic QED (NRQED) [6] where the constants can be determined order by order in $\alpha$, but many times is beyond our current technical abilities, like in the case of the chiral Lagrangian, which would require large lattice simulations with light dynamical quarks or yet-to-be-discovered alternative nonperturbative techniques with a good control on the mechanism of chiral symmetry breaking. In any case, if the number of constants is small enough, they can be phenomenologically obtained from available data and used later on to predict new results, as it is the case of the $\chi L$.

Pionium is a $\pi^{+} \pi^{-}$electromagnetic bound state of binding energy $\sim 2 \mathrm{keV}$ which decays strongly, basically to two

\footnotetext{
*Email address: dolors@ecm.ub.es

†Email address: soto@ecm.ub.es
}

$\pi^{0}$, with a width $\Gamma \sim 0.6 \mathrm{eV}$ [7]. Clearly a QCD based analysis of this system should better start with the $\chi L$. However, the $\chi L$ is a relativistic (manifestly Lorentz invariant) theory where electromagnetic bound state problems are difficult to handle (see [8,9] for direct approaches). Moreover, both the binding energy and the decay width are much smaller than the pion mass $\sim 140 \mathrm{MeV}$, which suggests that a nonrelativistic approach should be appropriated.

It is the aim of this work to present a nonrelativistic approach to pionium based on a series of EFTs which are obtained from the $\chi L$ coupled to electromagnetism after sequentially integrating out the various physical scales of the system until we reach the scale of the binding energy $\sim m \alpha^{2} / 4$. The first scale to be integrated out is the pion mass $m$. This produces a local nonrelativistic EFT for pion pairs near threshold coupled to electromagnetism, much in the same way as NRQED is obtained from QED $[6,10,11]$. The next relevant scale in the problem is the mass difference between charged and neutral pions $\Delta m \sim 5 \mathrm{MeV}$. Integrating out this scale produces a local EFT with only charged nonrelativistic pion fields coupled to electromagnetism. The next relevant scale is the typical relative momentum of pions in the bound state $m \alpha / 2 \sim 0.5 \mathrm{MeV}$ (soft). Integrating out this scale is, at lower orders of $\alpha$, equivalent to calculating the electromagnetic potential between the two charged pions. The calculations in the latter EFT reduce to quantum mechanical ones. The main advantage of this approach is that there are well defined counting rules at any stage of the calculation, so that the size of any neglected term is easy to estimate. This is particularly important in order to extract more accurate values for the parameters of the $\chi L$ from the improved measurement of the pionium decay width in the DIRAC experiment [3].

We distribute the paper as follows. In Sec. II we present the most general non-relativistic effective field theory for pion pairs near threshold. The constraints due to Lorentz invariance are implemented and the Lagrangian is reduced to its minimal form by local field redefinitions. In Sec. III the neutral pions are integrated out which gives rise to a nonrelativistic theory of charged pions interacting with the electromagnetic field. In Sec. IV we integrate out soft photons, which produce the electromagnetic potentials between the charged pions. In Sec. V we present the calculation of the bound state energies and decay widths. Section VI is devoted 
to the discussion of our results. In Appendix A we discuss the realization of Lorentz symmetry in nonrelativistic theories. In Appendix B we display the local field redefinitions and the various reshuffling of constants carried out along the paper. In Appendix $C$ we present a new way to regulate the Coulomb propagator in $D$ space dimensions.

\section{NONRELATIVISTIC LAGRANGIAN FOR PION PAIRS NEAR THRESHOLD}

At relative momentum much smaller than the pion mass a nonrelativistic description of pion pairs should be appropriated. In order to implement it, we shall write down a Lagrangian organized in powers of $1 / m$ in which any scale smaller than $m$ is treated perturbatively. For the problem at hand the next relevant energy and momentum scales are $\Delta m$ and $\sqrt{m \Delta m}$, its associated momentum, respectively. These scales are to be used to estimate the (maximum) size of each term.

The symmetries (exact and approximate) of the fundamental theory, namely the $\chi L$, must be incorporated. Let us consider first the internal symmetries. The $\chi L$ is approximately invariant under (nonlinear) chiral transformations, which are explicitly broken by the pion mass terms. Since the pion mass is a large parameter in the nonrelativistic Lagrangian, no algebraic constraints from chiral symmetry are expected to survive. All information about chiral symmetry will be hidden in the parameters of the Lagrangian. The only remaining approximate internal symmetry will be isospin, which is explicitly broken by $m_{u} \neq m_{d}$ and the e.m. interactions both at the quark and at the $\chi L$ level. The size of the explicit breaking may be estimated from $m_{\pi_{+}}-m_{\pi_{0}}$ $\sim 5 \mathrm{MeV}$ which is much smaller than the pion mass. Hence isospin symmetry is a good (approximate) symmetry for the nonrelativistic Lagrangian. In order to implement it we shall use the vector $\pi$

$$
\boldsymbol{\pi}=\left(\frac{\pi_{+}+\pi_{-}}{\sqrt{2}}, \frac{\pi_{-}-\pi_{+}}{\sqrt{2} i}, \pi_{0}\right),
$$

where $\pi_{+}, \pi_{-}$and $\pi_{0}$ annihilate positive, negative and neutral pions, respectively.

Concerning the space-time symmetries, Poincare invariance (including the discrete symmetries) must also be implemented in the nonrelativistic Lagrangian. The translational and rotational part of the Poincare group as well as the discrete symmetries are implemented in the standard way. The Lorentz subgroup requires the introduction of a nonlinear realization which is equivalent to impose the so called reparametrization invariance [12]. This is discussed in Appendix A. The outcome is relatively simple for spin zero fields. Consider a composite spin zero field made out of tensor products of $n \pi$ and $m \boldsymbol{\pi}^{\dagger}$. Define $w=n-m$ the weight of this field. If $w \neq 0$, all derivatives acting on this field must be introduced through the combination

$$
D=i \partial_{0}-\frac{1}{2 w m} \partial_{\mu} \partial^{\mu}
$$

If $w=0, \partial_{\mu}$ on this field can be introduced. The Lagrangian must have all the Lorentz indices contracted in a formally Lorentz invariant way and $D$ must be considered Lorentz invariant itself.

Having in mind the rules above, consider first the limit of exact isospin symmetry. We have

$$
\begin{aligned}
L= & L_{2}+L_{4}, \\
L_{2}= & L_{2}^{(0)}+L_{2}^{(1)}+\cdots, \\
L_{4}= & L_{4}^{(1 / 2)}+L_{4}^{(3 / 2)}+\cdots, \\
L_{2}^{(0)}= & \pi^{\dagger} D \boldsymbol{\pi}, \\
L_{2}^{(1)}= & \boldsymbol{\pi}^{\dagger} A_{0} D^{2} \boldsymbol{\pi}, \\
L_{4}^{(1 / 2)}= & B_{1}\left(\boldsymbol{\pi}^{\dagger} \boldsymbol{\pi}\right)^{2}+B_{2}(\boldsymbol{\pi} \boldsymbol{\pi})\left(\boldsymbol{\pi}^{\dagger} \boldsymbol{\pi}^{\dagger}\right), \\
L_{4}^{(3 / 2)}= & A_{1}(\boldsymbol{\pi} D \boldsymbol{\pi})\left(\boldsymbol{\pi}^{\dagger} \boldsymbol{\pi}^{\dagger}\right)+\text { H.c. } \\
& +A_{2}\left(\boldsymbol{\pi}^{\dagger} D \boldsymbol{\pi}\right)\left(\boldsymbol{\pi}^{\dagger} \boldsymbol{\pi}\right)+\text { H.c. } \\
& +A_{3}\left(\boldsymbol{\pi}^{\dagger} \boldsymbol{\pi}^{\dagger}\right) D(\boldsymbol{\pi} \boldsymbol{\pi}) \\
& +A_{4} \partial_{\mu}\left(\boldsymbol{\pi}^{\dagger} \boldsymbol{\pi}\right) \partial^{\mu}\left(\boldsymbol{\pi}^{\dagger} \boldsymbol{\pi}\right) \\
& +A_{5}\left(\boldsymbol{\pi}^{\dagger i} \boldsymbol{\pi}^{\dagger j}\right) D\left(\boldsymbol{\pi}^{i} \boldsymbol{\pi}^{j}\right) .
\end{aligned}
$$

Consider next the isospin breaking terms. These may be due to e.m. interactions at the quark level, e.m. interactions in the relativistic $\chi L$ and $m_{u} \neq m_{d}$. The electromagnetic interactions at quark level have an isospin invariant piece which is absorbed in the constants (2.3). The e.m. isospin breaking pieces, both at quark level and in the $\chi L$, are proportional to $T^{3}$, and so is the isospin breaking piece due to $m_{u} \neq m_{d}$. Hence, in order to incorporate isospin breaking effects in the nonrelativistic Lagrangian, it is enough to construct further invariants with the vectors $\mathbf{Q} \sim(0,0, e)$ and $\mathbf{M}$ $\sim\left(0,0, m_{u}-m_{d}\right)$, taking into account that $\mathbf{Q}$ must always appear in pairs. Although there is no extra difficulty in taking $\mathbf{M}$ into account, we shall ignore it here since, due to charge conjugation, it appears quadratically and turns out to be very small [13]. If we wish to recover these contributions at some point, we only have to remember that for any isospin breaking term proportional to $\alpha$, there is also an identical term proportional to $\left(m_{u}-m_{d}\right)^{2}$. Then the e.m. isospin breaking terms read

$$
\begin{gathered}
\Delta L=\Delta L_{2}+\Delta L_{4}, \\
\Delta L_{2}=\Delta L_{2}^{(0)}+\Delta L_{2}^{(1)}, \\
\Delta L_{4}=\Delta L_{4}^{(3 / 2),} \\
\Delta L_{2}^{(0)}=\delta_{1}\left(\boldsymbol{\pi}^{\dagger} \mathbf{Q}\right)(\mathbf{Q} \boldsymbol{\pi}), \\
\Delta L_{2}^{(1)}=\delta_{2}\left(\boldsymbol{\pi}^{\dagger} \mathbf{Q}\right) D(\mathbf{Q} \boldsymbol{\pi}),
\end{gathered}
$$




$$
\begin{aligned}
\Delta L_{4}^{(3 / 2)}= & C_{1}(\boldsymbol{\pi} \mathbf{Q})(\boldsymbol{\pi} \mathbf{Q})\left(\boldsymbol{\pi}^{\dagger} \boldsymbol{\pi}^{\dagger}\right)+\text { H.c. } \\
& +C_{2}(\boldsymbol{\pi} \mathbf{Q})\left(\boldsymbol{\pi}^{\dagger} \mathbf{Q}\right)\left(\boldsymbol{\pi}^{\dagger} \boldsymbol{\pi}\right) \\
& +C_{3}\left(\left(\boldsymbol{\pi}^{\dagger} \times \boldsymbol{\pi}\right) \cdot \mathbf{Q}\right)^{2} .
\end{aligned}
$$

Before going on, let us discuss the general structure of the constants $A_{i}, B_{i}, C_{i}$ and $\delta_{i}$ above. Let us call $Z$ to any such a constant and $z$ its dimension. Then the general form of $Z$ will be

$$
\begin{aligned}
Z= & m^{z}\left(a_{-1}+a_{0}\left(\frac{m^{2}}{4 \pi f^{2}}\right)+a_{1}\left(\frac{m^{2}}{4 \pi f^{2}}\right)^{2}+a_{2}\left(\frac{m^{2}}{4 \pi f^{2}}\right)^{3}+\cdots\right. \\
& \left.+b_{1} \alpha+\cdots+c_{1,1} \alpha\left(\frac{m^{2}}{4 \pi f^{2}}\right)+c_{1,2} \alpha\left(\frac{m^{2}}{4 \pi f^{2}}\right)^{2}+\cdots\right),
\end{aligned}
$$

where $f \sim 93 \mathrm{MeV}$ is the pion decay constant. The $a_{i}, i=$ $-1,0,1, \ldots$ stand for pure strong interaction contributions in chiral perturbation theory $(\chi \mathrm{PT})[5]$. It is interesting to notice that spontaneous chiral symmetry breaking implies $a_{-1}=0$ for $Z \neq A_{0}, \delta_{i}$. Indeed in the limit $f \rightarrow \infty$ (keeping $m$ constant) the pions in the $\chi L$ become free particles as far as the strong interactions is concerned. Hence, in this limit any EFT derived from the $\chi L$ must not contain strong interactions. Then the subscript $i=0,1, \ldots$ coincides with the number of loops at which the term $a_{i}$ receives contributions. We stopped at the number of loops which have been calculated so far [14]. $b_{i}, i=1,2, \ldots$ stand for purely electromagnetic contributions and $c_{i, j}, i, j=1,2, \ldots$ for mixed electromagnetic and strong contributions. We stop here at the orders which compare to the two loop purely strong contribution. $b_{1}$ may receive contributions from tree level annihilation graphs, $c_{1,1}$ from one loop graphs [15] and $c_{1,2}$ from two loop graphs yet to be calculated. For this discussion to apply to the constants $C_{i}$ and $\delta_{i}$ of the isospin breaking terms $\mathbf{Q}$ must be counted as a dimension one object. If $m_{u} \neq m_{d}$ was taken into account, additional series including $\left(m_{u}-m_{d}\right) / f$ would appear in Eq. (2.5).

The Lagrangian (2.3) and (2.4) contains higher time derivative terms. One can get rid of these terms by local field redefinitions. We can set $A_{0}=\delta_{2}=A_{1}=A_{2}=0$ by using local field redefinitions which maintain Lorentz symmetry explicit. However, the new Lagrangian still contains time derivatives beyond the expected $i \partial_{0}$. We can also get rid of the extra time derivatives by using again local field redefinitions, which cannot maintain Lorentz symmetry explicit anymore. The details of this are displayed in Appendix B. We finally obtain the Lagrangian in the so-called minimal form

$$
\begin{aligned}
L= & L_{2}+L_{4}, \\
L_{2}= & \boldsymbol{\pi}^{\dagger j}\left(\left(i \partial_{0}+\frac{\boldsymbol{\nabla}^{2}}{2 m}+\frac{\boldsymbol{\nabla}^{4}}{8 m^{3}}\right) \delta_{i j}+\left(1+\frac{\boldsymbol{\nabla}^{2}}{2 m^{2}}\right) \Delta m \frac{\mathbf{Q}^{i} \mathbf{Q}^{j}}{\mathbf{Q}^{2}}\right) \boldsymbol{\pi}^{i}, \\
L_{4}= & B_{1}\left(\boldsymbol{\pi}^{\dagger} \boldsymbol{\pi}\right)^{2}+B_{2}(\boldsymbol{\pi} \boldsymbol{\pi})\left(\boldsymbol{\pi}^{\dagger} \boldsymbol{\pi}^{\dagger}\right)+D_{1}\left(\boldsymbol{\pi}^{\dagger} \frac{\boldsymbol{\nabla}^{2}}{2 m} \boldsymbol{\pi}+\boldsymbol{\pi} \frac{\boldsymbol{\nabla}^{2}}{2 m} \boldsymbol{\pi}^{\dagger}\right)\left(\boldsymbol{\pi}^{\dagger} \boldsymbol{\pi}\right)+D_{2}\left(\left(\boldsymbol{\pi} \frac{\boldsymbol{\nabla}^{2}}{2 m} \boldsymbol{\pi}\right) \boldsymbol{\pi}^{\dagger} \boldsymbol{\pi}^{\dagger}+\boldsymbol{\pi} \boldsymbol{\pi}\left(\boldsymbol{\pi}^{\dagger} \frac{\boldsymbol{\nabla}^{2}}{2 m} \boldsymbol{\pi}^{\dagger}\right)\right) \\
& +2 A_{4}\left(\boldsymbol{\pi}^{\dagger} \boldsymbol{\pi}\right) \partial^{i} \boldsymbol{\pi}^{\dagger} \partial^{i} \boldsymbol{\pi}+C_{1}^{\prime}(\boldsymbol{\pi} \mathbf{Q})(\boldsymbol{\pi} \mathbf{Q})\left(\boldsymbol{\pi}^{\dagger} \boldsymbol{\pi}^{\dagger}\right)+\text { H.c. }+C_{2}^{\prime}(\boldsymbol{\pi} \mathbf{Q})\left(\boldsymbol{\pi}^{\dagger} \mathbf{Q}\right)\left(\boldsymbol{\pi}^{\dagger} \boldsymbol{\pi}\right)+C_{3}\left(\left(\boldsymbol{\pi}^{\dagger} \times \boldsymbol{\pi}\right) \cdot \mathbf{Q}\right)^{2} \\
& +\frac{A_{3}}{2}\left(\boldsymbol{\pi}^{\dagger} \boldsymbol{\pi}^{\dagger}\right) \frac{\boldsymbol{\nabla}^{2}}{2 m}(\boldsymbol{\pi} \boldsymbol{\pi})+\frac{A_{5}}{2}\left(\boldsymbol{\pi}^{\dagger i} \boldsymbol{\pi}^{\dagger j}\right) \frac{\boldsymbol{\nabla}^{2}}{2 m}\left(\boldsymbol{\pi}^{i} \boldsymbol{\pi}^{j}\right) .
\end{aligned}
$$

The new constants above are defined in formula (B8) of Appendix B. Lorentz symmetry guarantees that the bilinear terms have the standard form including relativistic corrections. It also relates $A_{3}$ and $A_{5}$ in the two last terms to the remaining constants (see Appendix B). Unfortunately, the latter relations have no practical consequences because the two last terms are proportional to the center of mass momentum and hence irrelevant to our problem. The zero charge sector in terms of the pion field reads

$$
\begin{aligned}
& L_{2}=\pi_{+}^{\dagger}\left(i \partial_{0}+\frac{\nabla^{2}}{2 m}+\frac{\nabla^{4}}{8 m^{3}}\right) \pi_{+}+\pi_{-}^{\dagger}\left(i \partial_{0}+\frac{\nabla^{2}}{2 m}+\frac{\nabla^{4}}{8 m^{3}}\right) \pi_{-}+\pi_{0}^{\dagger}\left(i \partial_{0}+\Delta m+\frac{\nabla^{2}}{2 m}+\Delta m \frac{\nabla^{2}}{2 m^{2}}+\frac{\nabla^{4}}{8 m^{3}}\right) \pi_{0}, \\
L_{4}= & R_{00} \pi_{0}^{\dagger} \pi_{0}^{\dagger} \pi_{0} \pi_{0}+R_{c c} \pi_{+}^{\dagger} \pi_{-}^{\dagger} \pi_{+} \pi_{-}+\left(R_{0 c} \pi_{0}^{\dagger} \pi_{0}^{\dagger} \pi_{+} \pi_{-}+\text {H.c. }\right)+S_{00}\left(\pi_{0}^{\dagger} \pi_{0}^{\dagger} \pi_{0} \nabla^{2} \pi_{0}+\text { H.c. }\right)+S_{c c}\left(\pi _ { + } ^ { \dagger } \pi _ { - } ^ { \dagger } \left(\pi_{+} \nabla^{2} \pi_{-}\right.\right. \\
& \left.\left.+\pi_{-} \nabla^{2} \pi_{+}\right)+ \text {H.c }\right)+S_{0 c}\left(\pi_{0}^{\dagger} \pi_{0}^{\dagger}\left(\pi_{+} \nabla^{2} \pi_{-}+\pi_{-} \nabla^{2} \pi_{+}\right)+2 \pi_{+}^{\dagger} \pi_{-}^{\dagger} \pi_{0} \nabla^{2} \pi_{0}+\text { H.c. }\right)+P_{00} \pi_{0}^{\dagger} \partial_{i} \pi_{0}^{\dagger} \pi_{0} \partial_{i} \pi_{0} \\
& +P_{c c}\left(\pi_{+}^{\dagger} \partial_{i} \pi_{-}^{\dagger} \pi_{+} \partial_{i} \pi_{-}+\pi_{-}^{\dagger} \partial_{i} \pi_{+}^{\dagger} \pi_{-} \partial_{i} \pi_{+}\right) .
\end{aligned}
$$

The new constants above are defined in formula (B9) of Appendix B. Notice that since the origin of energies appears to be at the two charged pion threshold, the neutral pion shows a negative energy gap $-\Delta m<0$. Notice also that the terms in the bilinear neutral pion Lagrangian can be combined into the standard form: 


$$
\left(i \partial_{0}+\Delta m+\frac{\boldsymbol{\nabla}^{2}}{2 m}+\Delta m \frac{\boldsymbol{\nabla}^{2}}{2 m^{2}}+\frac{\boldsymbol{\nabla}^{4}}{8 m^{3}}\right) \sim\left(i \partial_{0}+\Delta m+\frac{\boldsymbol{\nabla}^{2}}{2(m-\Delta m)}+\frac{\boldsymbol{\nabla}^{4}}{8(m-\Delta m)^{3}}\right) .
$$

Nevertheless, in order to keep the expansion systematic we shall not use the expression above.

The coupling to e.m. fields is done by promoting normal derivatives to covariant derivatives. None of the possible nonminimal couplings contributes at the order we are interested in and we will ignore them.

Before closing this section let us remark that we have assumed that the Lagrangian (2.3) and (2.4) is Hermitian. This is correct at the order we are interesting in. However, in general the Hermiticity constraint must be relaxed. This is due to the fact that the $\pi^{+} \pi^{-}$atom may decay into degrees of freedom which do not appear in the nonrelativistic Lagrangian, for instance to hard photons or hard electronpositron pairs. The non-Hermitian pieces would be obtained in the matching to the $\chi L$ at the same time as the Hermitian ones, as it happens in NRQED $[10,11,18]$.

\section{INTEGRATING OUT THE SCALE $\Delta m$}

Since $\Delta m \gg m \alpha^{2} / 4$ it is appropriated to integrate out this scale before tackling the e.m. bound state problem. This represents the main advantage of our approach with respect to the nonrelativistic proposals $[2,17]$. The integration of neutral pions can be easily achieved by matching four point off-shell Green functions of the Lagrangian above to a nonrelativistic Lagrangian where the neutral pions have been removed:

$$
\begin{aligned}
L^{\prime}= & \pi_{+}^{\dagger}\left(i D_{0}+\frac{\mathbf{D}^{2}}{2 m}\right) \pi_{+}+\pi_{-}^{\dagger}\left(i D_{0}+\frac{\mathbf{D}^{2}}{2 m}\right) \pi_{-} \\
& +R_{c c}^{\prime} \pi_{+}^{\dagger} \pi_{-}^{\dagger} \pi_{+} \pi_{-}+P^{\prime} \pi_{+}^{\dagger} \pi_{-}^{\dagger} i \partial_{0} \pi_{+} \pi_{-}
\end{aligned}
$$

Since the $\pi^{0}$ energy gap is negative, the integration will produce imaginary parts in $R_{c c}^{\prime}$ and $P^{\prime}$. By calculating the
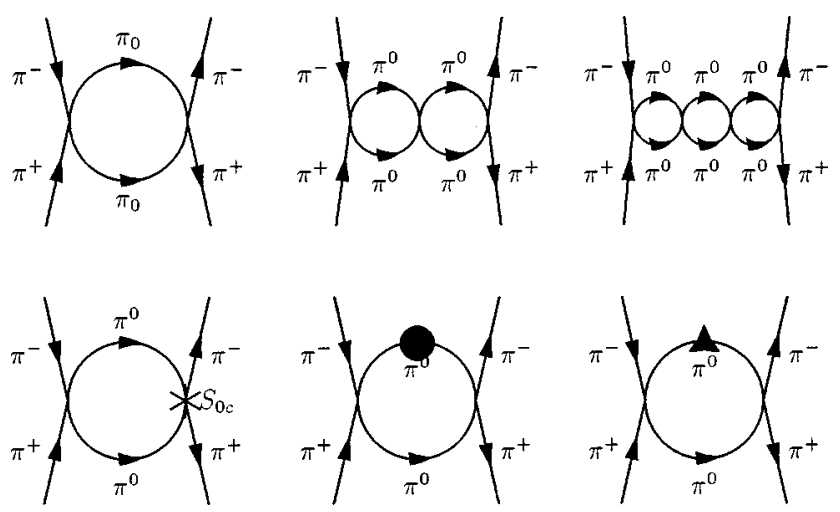

FIG. 1. Diagrams contributing to the matching between $L$ and $L^{\prime}$ up to corrections $O\left((\Delta m / m)^{2}\right)$. The bullet and triangle insertions in the neutral pion propagator correspond to relativistic corrections due to $\nabla^{4} / 8 m^{3}$ and $\Delta m \nabla^{2} / 2 m^{2}$, respectively. diagrams in Fig. 1 in dimensional regularization (DR) we obtain

$$
\begin{aligned}
R_{c c}^{\prime}= & R_{c c}-\left|R_{0 c}\right|^{2} R_{00}\left(\frac{m s}{2 \pi}\right)^{2}+i\left|R_{0 c}\right|^{2} \frac{m s}{2 \pi}\left(1+\frac{5}{8} \frac{s^{2}}{m^{2}}-\frac{3}{4} \frac{s^{2}}{m^{2}}\right. \\
& \left.-\left(\frac{R_{00} m s}{2 \pi}\right)^{2}-\frac{2 S_{0 c}\left(R_{0 c}+R_{0 c}^{*}\right) s^{2}}{\left|R_{0 c}\right|^{2}}\right) \\
P^{\prime}= & i\left|R_{0 c}\right|^{2} \frac{m^{2}}{4 \pi s},
\end{aligned}
$$

where $s=\sqrt{2 m \Delta m} \cdot R_{c c}^{\prime}$ and $P^{\prime}$ contain the leading corrections in $\Delta m / m$ and $m \alpha^{2} / 4 \Delta m$, respectively.

The electromagnetic contributions to $L^{\prime}$ coming from the energy scale $\Delta m$ are negligible, as well as the relativistic corrections $\sim \nabla^{4} / 8 m^{3}$ to the charge pions and the terms $P_{c c}$ and $S_{c c}$ in Eq. (2.7).

\section{INTEGRATING OUT THE SCALE $m \alpha$}

The Lagrangian in the previous section is almost identical to NRQED (for spin zero particles) plus small local interactions. In Refs. [18] it was shown that we can integrate out next dynamical scale, namely, $m \alpha / 2$ in NRQED obtaining a further effective theory called potential NRQED (pNRQED) which contains the usual potential terms and only the ultrasoft degrees of freedom $\left(\sim m \alpha^{2} / 4\right)$ remain dynamical. We shall do the same here. The (maximum) size of each term in Eq. (3.1) is obtained by assigning $m \alpha$ to any scale which is not explicit. In fact, since we are only interested in $O(\alpha)$ corrections, only the Coulomb potential seems to be important, since the tranverse photons give rise to $O\left(\alpha^{2}\right)$ corrections. However, as pointed out in Ref. [19], below the pion threshold there are further light degrees of freedom apart from the photon. In particular, the electron mass $m_{e} \sim m \alpha / 2$ and hence it must be integrated out here. This gives rise to a potential term which is only $O(\alpha)$ suppressed with respect to the Coulomb one. By calculating the diagrams in Fig. 2 we obtain

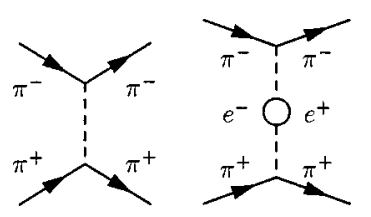

FIG. 2. Diagrams contributing to the matching between $L^{\prime}$ and $L^{\prime \prime}$ up to corrections $O\left(\alpha^{2}\right)$. Dashed lines are longitudinal photon propagators in the Coulomb gauge. 


$$
\begin{aligned}
& L^{\prime \prime}= \pi_{+}^{\dagger}(\mathbf{x}, t)\left(i \partial_{0}+\frac{\boldsymbol{\nabla}^{2}}{2 m}\right) \pi_{+}(\mathbf{x}, t)+\pi_{-}^{\dagger}(\mathbf{x}, t)\left(i \partial_{0}+\frac{\nabla^{2}}{2 m}\right) \\
& \times \pi_{-}(\mathbf{x}, t)+R_{c c}^{\prime}\left(\pi_{+}^{\dagger} \pi_{-}^{\dagger} \pi_{+} \pi_{-}\right)(\mathbf{x}, t) \\
&+P^{\prime}\left(\pi_{+}^{\dagger} \pi_{-}^{\dagger}\right)(\mathbf{x}, t) i \partial_{0}\left(\pi_{+} \pi_{-}\right)(\mathbf{x}, t)-\int d^{3} \mathbf{y}\left(\pi_{+}^{\dagger} \pi_{+}\right) \\
& \times(\mathbf{x}, t)\left(V_{0}(|\mathbf{x}-\mathbf{y}|)+V_{1}(|\mathbf{x}-\mathbf{y}|)\right)\left(\pi_{-}^{\dagger} \pi_{-}\right)(\mathbf{y}, t), \\
& V_{0}(|\mathbf{x}-\mathbf{y}|)=-\frac{\alpha}{|\mathbf{x}-\mathbf{y}|}, \\
& V_{1}(|\mathbf{x}-\mathbf{y}|)=\int \frac{d^{3} \mathbf{k}}{(2 \pi)^{3}} V_{v p c}(\mathbf{k}) e^{i(\mathbf{x}-\mathbf{y}) \mathbf{k},}
\end{aligned}
$$

where $V_{v p c}(\mathbf{k})$ is given in formula (10) of Ref. [19]. The Lagrangian above contains no further degree of freedom than the nonrelativistic charged pions and hence it is totally equivalent to standard quantum mechanics. We prefer to stay within the Lagrangian formalism and use the $\pi^{-} \pi^{+}$wave function field $\phi(\mathbf{x}, \mathbf{X}, t)$, where $\mathbf{x}$ and $\mathbf{X}$ are the relative and center of mass coordinates, respectively, as introduced in [18]:

$$
\begin{aligned}
L^{\prime \prime}= & \phi^{\dagger}(\mathbf{x}, \mathbf{X}, t)\left(i \partial_{0}+\frac{\nabla^{2}}{m}-V_{0}(|\mathbf{x}|)-V_{1}(|\mathbf{x}|)+R_{c c}^{\prime} \delta(\mathbf{x})\right. \\
& \left.+P^{\prime} \delta(\mathbf{x}) i \partial_{0}\right) \phi(\mathbf{x}, \mathbf{X}, t) .
\end{aligned}
$$

The center of mass kinetic term has been dropped.

\section{QUANTUM MECHANICAL CALCULATION}

In order to calculate the corrections to the energy levels and decay width we shall consider the propagator of Eq. (4.3) and identify its pole. At the order we are interested in only the diagrams in Fig. 3 contribute.

The diagrams in the first line of Fig. 3 correspond to first order perturbation theory and can be easily evaluated. They give rise to

$$
\begin{aligned}
& \delta_{R_{c c}^{\prime}} E_{n}^{(1)}=-\operatorname{Re}\left(R_{c c}^{\prime}\right)\left|\Psi_{n}(\mathbf{0})\right|^{2}, \\
& \delta_{R_{c c}^{\prime}} \Gamma_{n}^{(1)}=2 \operatorname{Im}\left(R_{c c}^{\prime}\right)\left|\Psi_{n}(\mathbf{0})\right|^{2},
\end{aligned}
$$

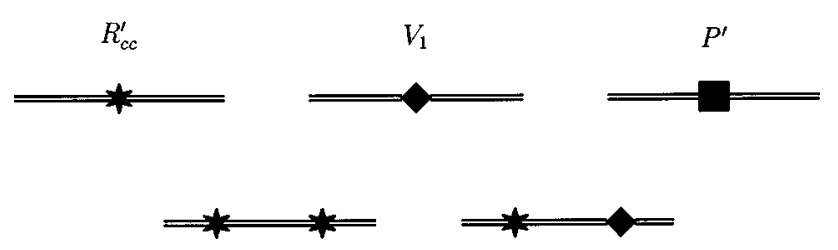

FIG. 3. Diagrams contributing to the leading order corrections in $\Delta m / m, \alpha$ and $m \alpha^{2} / \Delta m$ to the energy and decay width. The double line is the Coulomb propagator of the $\pi^{+} \pi^{-}$pair.

$$
\begin{gathered}
\delta_{P^{\prime}} E_{n}^{(1)}=0, \quad \delta_{P^{\prime}} \Gamma_{n}^{(1)}=-\operatorname{Im}\left(P^{\prime}\right)\left|\Psi_{n}(\mathbf{0})\right|^{2}\left(\frac{m \alpha^{2}}{2 n^{2}}\right), \\
\delta_{V_{1}} E_{1}^{(1)}=\frac{11 m \alpha^{3}}{18 \pi}\left(1-\frac{9 \pi}{22} \xi+\frac{12}{11} \xi^{2}-\frac{6 \pi}{11} \xi^{3}\right. \\
\left.-\frac{3\left(2-\xi^{2}-4 \xi^{4}\right)}{11 \sqrt{\xi^{2}-1}} \tan ^{-1} \sqrt{\xi^{2}-1}\right), \\
\xi:=\frac{2 m_{e}}{m \alpha}, \quad \delta_{V_{1}} \Gamma_{n}^{(1)}=0
\end{gathered}
$$

where $\Psi_{n}(\mathbf{x})$ is the Coulomb wave function.

The diagrams in the second line of Fig. 3 correspond to second order perturbation theory and are not so easily calculated. The second diagram gives a finite contribution $\delta_{V_{1}} \Gamma_{n}^{(2)}$ which for the ground state has been evaluated numerically in [19]. The first diagram has also been considered before [17]. However, since it is UV divergent a suitable regularization and renormalization scheme must be specified. The subtraction point dependence of the result will eventually cancel against the subtraction point dependences in the matching coefficients. The matching coefficients are to be found by matching the $\chi L$ with e.m. interactions with Eqs. (2.3) and (2.4) at the desired order of $\chi \mathrm{PT}$ and $\alpha$. The matching calculation is most efficiently done at threshold using DR and MS (or $\overline{\mathrm{MS}}$ ) scheme for both UV and IR divergences [11]. This requires to use the same regularization and renormalization scheme when calculating in the effective theory. We have calculated in Appendix C the first diagram using DR and MS scheme so that our results can be readily applied once the above mentioned matching calculation is carried out. We obtain

$$
\begin{aligned}
& \delta_{R_{c c}^{\prime}} E_{n}^{(2)}=\operatorname{Re}\left(R_{c c}^{\prime}{ }^{2}\right) \frac{m^{2} \alpha \Delta_{n}}{4 \pi}\left|\Psi_{n}(\mathbf{0})\right|^{2}, \\
& \delta_{R_{c c}^{\prime}} \Gamma_{n}^{(2)}=-2 \operatorname{Im}\left(R_{c c}^{\prime}{ }^{2}\right) \frac{m^{2} \alpha \Delta_{n}}{4 \pi}\left|\Psi_{n}(\mathbf{0})\right|^{2},
\end{aligned}
$$

where $\Delta_{n}$ is given in formula (C12) of Appendix C. Notice that Eqs. (5.1)-(5.5) hold at any order of $\chi \mathrm{PT}$, since we have made no use of the $m^{2} / 4 \pi f^{2}$ expansion in order to derive them from Eq. (2.7).

Putting all this together, our final expressions for the energy and the decay width read

$$
\begin{gathered}
E_{n}=-\frac{m \alpha^{2}}{4 n^{2}}-\frac{\left|\Psi_{n}(\mathbf{0})\right|^{2}}{2 f^{2}}+\delta_{V_{1}} E_{n}^{(1)} \\
\Gamma_{n}=\Gamma_{n}^{(0)}\left(1+\Delta_{\chi P T}+\frac{5 \Delta m}{12 m}-\frac{m \alpha^{2}}{16 \Delta m n^{2}}-\frac{m^{2} \alpha \Delta_{n}}{4 \pi f^{2}}\right) \\
+\delta_{V_{1}} \Gamma_{n}^{(2)}
\end{gathered}
$$




$$
\Gamma_{n}^{(0)}=\frac{9 m \sqrt{2 m \Delta m}}{64 \pi f^{4}}\left|\Psi_{n}(\mathbf{0})\right|^{2},
$$

where we have substituted $R_{00}, R_{c c}, R_{0 c}$ and $S_{0 c}$ by their tree level values in the corrections

$$
\begin{aligned}
R_{00} & \sim \frac{1}{16 f^{2}}, \\
R_{0 c} & \sim \frac{3}{8 f^{2}}, \\
R_{c c} & \sim \frac{1}{2 f^{2}}, \\
S_{0 c} & \sim-\frac{1}{32 m^{2} f^{2}}
\end{aligned}
$$

and defined

$$
\left|R_{0 c}\right|^{2}=\left(\frac{3}{8 f^{2}}\right)^{2}\left(1+\Delta_{\chi P T}\right) .
$$

We have also dropped terms proportional to $R_{00}$ in Eq. (3.2) because they are suppressed by extra factors of $m^{2} / 4 \pi f^{2}$. $\Delta_{\chi P T}$ summarizes all the contributions to $\left|R_{0 c}\right|^{2}$ beyond the one at tree level in the isospin symmetric limit, in particular those from pion and photon loops in the $\chi L$. The structure of $\Delta_{\chi P T}$ is then inherited from $\left|R_{0 c}\right|^{2}$ and follows easily from Eq. (2.5). $\delta_{V_{1}} E_{n}^{(1)}$ is given for $n=1$ in Eq. (5.3) and $\delta_{V_{1}} \Gamma_{n}^{(2)}$ is only known for $n=1$ [19]:

$$
\delta_{V_{1}} \Gamma_{1}^{(2)} \sim 0.4298 \alpha \Gamma_{1}^{(0)} .
$$

Formulas (5.6) and (5.7) are exact up to next to leading order in $\alpha, \Delta m / m$ and $m \alpha^{2} / \Delta m$, except for the optional substitutions mentioned above. Notice that only $R_{0 c}$ is needed beyond tree level $\left(\Delta_{\chi P T}\right)$. In $\Delta_{\chi P T}$ there should be a contribution $\sim \log m / \mu$ which cancels the $\mu$ dependence in $\Delta_{n}$. This would arise from a two loop calculation involving photons which has not been carried out yet. It requires the evaluation of diagrams analogous to Fig. 4 but with relativistic pion propagators.

\section{DISCUSSION}

We have presented an approach to pionium which consists of separating the various dynamical scales involved in the problem by using effective field theory techniques. The main advantage of this approach is, apart from its simplicity, that error estimates can be carried out very easily. A few remarks concerning other approaches are in order. First of all, relativistic approaches [8,9], apart from being technically more involved, have all the scales in the problem entangled which makes very difficult to estimate errors or to gauge the size of a given diagram. We would like to emphasize that Lorentz symmetry, even though it is not linearly realized, it is implemented in our approach to the required order. Several nonrelativistic approaches have appeared in the literature addressing particular aspects of the problem $[16,17,19]$. Our analysis shows that a coupled channel approach to pionium [17] is unnecessary because the $\Delta m$ is much larger than the bound state energy. It also shows that, although it is technically possible (trivial in fact) to make a resummation of bubble diagrams in the manner of Lipmann-Schwinger, it does not make much sense doing it since there are higher derivative terms in the effective Lagrangian, which have been neglected, which would give rise to contributions of the same order. In a way, our approach implements the remark of [16] related to the fact that neutral pion loops give rise to important contributions in the nonrelativistic regime. We have supplemented this remark with a full theoretical framework and with relativistic corrections of the same order which had been overlooked.

Let us next discuss how our results compare to the Deser formula [1], and how they are related to the strong scattering lengths (SSL). In fact the concept of SSL itself requires some qualifications. In order to be precise, let us define three kinds of SSL: (i) the QCD SSL (QCDSSL) as those calculated from QCD, (ii) the chiral Lagrangian SSL ( $\chi \mathrm{LSSL}$ ) as those calculated from the $\chi L$ [14], and (iii) the nonrelativistic SSL (NRSSL) as those calculated from nonrelativistic potential models or effective theories of the strong interactions $[2,16,17]$. At present the QCDSSL cannot be calculated reliably and hence they are not useful to compare with experimental results. The $\chi \mathrm{LSSL}$ are useful to compare with particle physics data at energies of the order of the pion mass. However, even if electromagnetism is not included in the $\chi L$, the $\chi$ LSSL obtained from those experiments contain some electromagnetic contributions since it is not possible to switch off the electromagnetic interaction between quarks. Hence, strictly speaking the $\chi$ LSSL are already not purely strong. The NRSSL should be useful to handle nuclear physics data of pion scattering at nonrelativistic energies near threshold. It is within this framework that Deser type formulas, which relate the decay width of pionium to the pion SSL are derived. However, such kind of scattering data is almost nonexisting, and the NRSSL have a limited practical interest unless one is able to relate them to the $\chi$ LSSL. Even if electromagnetism is not included in our potential model or nonrelativistic effective theory, the NRSSL contain, in addition to the unremovable electromagnetic contributions at the quark level, those due to photons at (relativistic) scales of the order of the pion mass. This is particularly transparent in our formulation [see Eq. (2.5)]. Once the matching between the $\chi L$ and the Lagrangian (2.3), (2.4) is carried out, the relation between the NRSSL and the $\chi$ LSSL will become available. For the moment, the best we can do is to rewrite Eqs. (5.7), (5.8) in terms of NRSSL and compare with Deser type formulas.

From Eq. (2.7) we obtain

$$
\begin{aligned}
\operatorname{Re}(A(+- & \rightarrow 00))\left.\right|_{\text {thres }} \\
= & 2 \operatorname{Re}\left(R_{0 c}\right)\left(1-R_{00}^{2}\left(\frac{m s}{2 \pi}\right)^{2}-\frac{2 S_{0 c} s^{2}}{\operatorname{Re}\left(R_{0 c}\right)}\right) \\
& -2 \operatorname{Im}\left(R_{0 c}\right) R_{00} \frac{m s}{2 \pi} \\
\sim & 2 \operatorname{Re}\left(R_{0 c}\right)\left(1+\frac{\Delta m}{3 m}\right) .
\end{aligned}
$$


Recall that $\operatorname{Im}\left(R_{0 c}\right)$ is proportional to an isospin breaking term $\sim \Delta m / m, \alpha$ [see Eq. (B9)]. Hence we can neglect the term proportional to $\operatorname{Im}\left(R_{0 c}\right)$ above and $\operatorname{Im}\left(R_{0 c}\right)^{2}$ below. We have also neglected the term proportional to $R_{00}^{2}$ since it is suppressed by an extra factor $\left(\mathrm{m}^{2} / 4 \pi f^{2}\right)^{2}$. This allows us to solve $\left|R_{0 c}\right|^{2}$ in terms of the corresponding NRSSL $a_{0 c}$ :

$$
\begin{gathered}
\operatorname{Re}\left(\left.A(+-\rightarrow 00)\right|_{\text {thres }}\right):=a_{0 c} \frac{8 \pi}{m^{2}}, \\
\left|R_{0 c}\right|^{2} \sim a_{0 c}^{2} \frac{16 \pi^{2}}{m^{4}}\left(1-\frac{2 \Delta m}{3 m}\right) .
\end{gathered}
$$

Then we can rewrite Eq. (5.7) as

$$
\begin{aligned}
\Gamma_{n}= & \frac{16 \pi \sqrt{2 m \Delta m}}{m^{3}} a_{0 c}^{2}\left|\Psi_{n}(\mathbf{0})\right|^{2}\left(1-\frac{\Delta m}{4 m}-\frac{m \alpha^{2}}{16 \Delta m n^{2}}\right. \\
& \left.-\frac{m^{2} \alpha \Delta_{n}}{4 \pi f^{2}}\right)+\delta_{V_{1}} \Gamma_{n}^{(2)},
\end{aligned}
$$

where the Deser formula is recovered upon neglecting the corrections $O\left(\Delta m / m, m \alpha^{2} / 4 \Delta m, \alpha\right)$. Let us emphasize again that the NRSSL $a_{0 c}$ above corresponds to the one used in nonrelativistic potential models [2] or effective theories $[16,17]$ but not the $\chi$ LSSL to be used in the $\chi L$. The formula above holds up to corrections $O\left((\Delta m / m)^{2}\right.$, $\left(m \alpha^{2} / \Delta m\right)^{2}, \alpha^{2}, \alpha\left(m^{2} / 4 \pi f^{2}\right)^{2},\left(m^{2} / 4 \pi f^{2}\right)^{2}(\Delta m / m), \alpha\left(m^{2} /\right.$ $\left.\left.4 \pi f^{2}\right) \sqrt{\Delta m / m},\left(m^{2} / 4 \pi f^{2}\right)(\Delta m / m)^{3 / 2}\right)$.

On the technical side we have worked out a new method to calculate the Coulomb propagator $G_{c}(0,0 ; E)$ in dimensional regularization (DR). The expressions for $G_{c}(0,0 ; E)$ when $E \rightarrow E_{n}$ are easily obtained for any $n$. Using DR here it is not just a matter of taste. Eventually a two loop matching calculation is to be done in order to extract the parameters of the Chiral Lagrangian from the pionium width. These kind of calculations are only efficiently done in the DR. Since the matching coefficients depend on the renormalization scheme, it is important to have our calculation in DR in order to be able to use the outcome of such a matching calculation straight away.

While this paper was being written up Ref. [20] appeared which deals with the same problem by similar techniques. If we neglect $\delta_{V_{1}} \Gamma_{1}^{(2)}$ (vacuum polarization), $\Gamma_{1}$ obtained from Eqs. (5.1), (5.2) and (5.5) coincides with that given in for- mulas (10) and (11) of [20], upon expanding the latter in $\Delta m / m$ and $m \alpha^{2} / 4 \Delta m$ and keeping terms up to next to leading order. Upon completion of this work Ref. [21] also appeared, where the relativistic corrections corresponding to the two last diagrams of Fig. 1 were calculated. The imaginary part of formula (3.2) agrees with the results of $[16,21]$ before any particular value is taken for the parameters in Eq. (2.7).

\section{ACKNOWLEDGMENTS}

J.S. acknowledges discussions with A. Gall, J. Gasser, V. E. Lyubovitskij, and A. Rusetsky. D.E. has benefited from financial support of the Spanish MEC FPI. Financial support from the CICYT (Spain), contract AEN98-0431, and the CIRIT (Catalonia), contract 1998SGR 00026 is also acknowledged.

\section{APPENDIX A: LORENTZ SYMMETRY IN NONRELATIVISTIC EFFECTIVE THEORIES}

Consider $\phi(x)$ a relativistic spin zero field and its partition function

$$
Z(J)=\int D \phi e^{i\left(S(\phi)+\int d^{4} x J(x) \phi(x)\right)} .
$$

If $S$ is Lorentz invariant then

$$
Z(J)=Z\left(J^{\prime}\right), \quad J^{\prime}(x)=J\left(\Lambda^{-1} x\right) .
$$

In the nonrelativistic regime we only need a subset of $J s$ which generate Green functions with the external legs almost on shell. These may be chosen as

$$
J(x)=\sqrt{2 m}\left(e^{-i m x^{0}} J_{h}(x)+e^{i m x^{0}} J_{h}^{\dagger}(x)\right),
$$

where $m$ is the mass of $\phi$ and $J_{h}(x)$ is slowly varying (i.e., contains energy and momentum much smaller than $m$ ). From Eqs. (A2) and (A3) one easily finds that for Lorentz transformations close to the identity

$$
J_{h}(x) \rightarrow J_{h}^{\prime}(x)=e^{-i m\left(\Lambda^{-1}-1\right)^{0}{ }^{x}{ }^{\mu}} J_{h}\left(\Lambda^{-1} x\right) .
$$

In the nonrelativistic regime $Z(J)$ can be approximated to the desired order of accuracy by

$$
Z(J) \sim Z_{N R}\left(J_{h}, J_{h}^{\dagger}\right)=\int D h d h^{\dagger} e^{i\left(S_{N R}\left(h, h^{\dagger}\right)+\int d^{4} x\left[h^{\dagger}(x) J_{h}(x)+J_{h}^{\dagger}(x) h(x)\right]\right)} .
$$

Then $Z_{N R}\left(J_{h}, J_{h}^{\dagger}\right)$ must be invariant under the transformation (A4). Invariance of the terms coupled to the sources implies the following transformations for $h(x)$ :

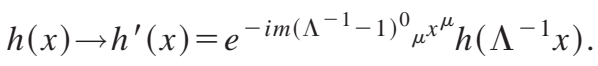

Hence $S_{N R}\left(h, h^{\dagger}\right)$ must be constructed in such a way that it is invariant under Eq. (A6). In order to do so, notice first of all that $\partial_{\mu} h(x)$ does not transform in a way similar to $h(x)$. We would like to introduce a kind of covariant derivative. The following operator appears to be a successful candidate: 


$$
D=i \partial_{0}-\frac{\partial_{\mu} \partial^{\mu}}{2 m}
$$

We have under Lorentz transformations

$$
D h(x) \rightarrow e^{-i m\left(\Lambda^{-1}-1\right)^{0}{ }_{\mu} x^{\mu}}\left(D+i\left(\Lambda^{-1}-1\right)^{0}{ }_{\mu} \partial^{\mu}\right) h\left(\Lambda^{-1} x\right),
$$

which upon the change $x \rightarrow \Lambda x$ becomes

$$
D h(x) \rightarrow e^{-i m(1-\Lambda)^{0}}{ }_{\mu^{x}}{ }^{\mu} \operatorname{Dh}(x) .
$$

Analogously, if we have $C_{w}(x)=\left(h^{\dagger}(x)\right)^{m}(h(x))^{n}, w=n$ $-m$, we may define for $w \neq 0$ a generalization of Eq. (A7)

$$
D=i \partial_{0}-\frac{\partial_{\mu} \partial^{\mu}}{2 w m}
$$

Then $D^{k} C_{w}(x)$ has the same transformation properties as $C_{w}(x)$. We call $w$ the weight of the composite field $C_{w}(x)$. If $w=0$ then $\partial_{\mu} C_{0}(x)$ transforms as a usual Lorentz vector.

From the discussion above the following rules can be inferred in order to built a Lorentz invariant nonrelativistic effective theory for spin zero particles: (i) Write down all possible terms in the particle sector we are interested in with weight zero and no derivatives up to the desired order; (ii) for each term, which is not already of the higher relevant order, insert $D s$ or $\partial_{\mu} s$ in all possible ways. All $\mu$ indices coming from the $\partial_{\mu}$ must be contracted in a Lorentz invariant way.

Applying the rules above we obtain the Lagrangians (2.3) and (2.4). Recall also that for the particular case we are interested in the (minimal) suppression of $D$ is $\Delta m / m$ whereas the (minimal) suppression of $\partial_{\mu}$ is $\sqrt{\Delta m / m}$.

Finally, let us mention that for practical purposes the rules that we have obtained are identical to those derived from the so called reparametrization invariance [12] (see also [23]). Hence, it should be clear that reparametrization invariance is nothing but a way to implement Lorentz symmetry in a nonrelativistic theory. We believe that this point is important and has not been sufficiently stressed in the literature.

\section{APPENDIX B: LOCAL FIELD REDEFINITIONS}

The Lagrangian given in formulas (2.3) and (2.4) contains higher time derivative terms whereas the usual nonrelativistic Lagrangians contain only a time derivative in the bilinear terms of each field. The latter is known as the minimal form of the Lagrangian. In this Appendix we display the local field redefinitions which bring the Lagrangian (2.3) and (2.4) to its minimal form. Let us only mention that local field redefinitions exploit the freedom we have in field theory to choose the interpolating field we wish, and refer the interested reader to the literature on the subject $[12,22,24,25,26]$. The price we pay for having the Lagrangian in its minimal form is that Lorentz symmetry (reparametrization invariance) will not be explicit anymore. The constraints given by Lorentz symmetry will reduce to nontrivial relations between the parameters of the Lagrangian in its minimal form.

We are retaining corrections up to the relative order
$(\Delta m / m)^{3 / 2}$. In order to reduce our operator basis, we will take advantage of the fact that local field redefinitions can also be organized in powers of $\Delta \mathrm{m} / \mathrm{m}$. The induced terms beyond the desired order as well as the terms which do not contribute to the two particle sector (six pion terms and beyond) will be neglected.

Let us first consider local field redefinitions which keep Lorentz symmetry explicit. We can get rid of the $A_{0}$ and $\delta_{2}$ terms in Eqs. (2.3) and (2.4) by

$$
\boldsymbol{\pi}^{i} \mapsto\left(\left(1-\frac{D A_{0}}{2}\right) \delta^{i j} \boldsymbol{\pi}^{j}+\left(\frac{\delta_{1} A_{0} \mathbf{Q}^{i} \mathbf{Q}^{j}}{2}-\frac{\delta_{2} \mathbf{Q}^{i} \mathbf{Q}^{j}}{2}\right)\right) \boldsymbol{\pi}^{j} .
$$

The bilinear terms become

$$
L_{2}+\Delta L_{2}=\boldsymbol{\pi}^{\dagger} D \boldsymbol{\pi}+\boldsymbol{\pi}^{\dagger i} \delta_{1} \mathbf{Q}^{i} \mathbf{Q}^{j}\left(1+\left(\delta_{1} A_{0}-\delta_{2}\right) \mathbf{Q}^{2}\right) \pi^{\mathbf{j}}
$$

and the following constants of the four pion terms get modified:

$$
\begin{aligned}
& A_{1} \rightarrow A_{1}^{\prime}=A_{1}-A_{0} B_{2}, \\
& A_{2} \rightarrow A_{2}^{\prime}=A_{2}-A_{0} B_{1}, \\
& C_{1} \rightarrow C_{1}^{\prime \prime}=C_{1}-\left(\delta_{2}-\delta_{1} A_{0}\right) B_{2}, \\
& C_{2} \rightarrow C_{2}^{\prime \prime}=C_{2}-2\left(\delta_{2}-\delta_{1} A_{0}\right) B_{1} .
\end{aligned}
$$

We can also get rid of the $A_{1}^{\prime}$ and $A_{2}^{\prime}$ keeping Lorentz invariance by making

$$
\pi \mapsto \pi-A_{2}^{\prime *} \boldsymbol{\pi}\left(\boldsymbol{\pi}^{\dagger} \boldsymbol{\pi}\right)-A_{1}^{\prime *} \boldsymbol{\pi}^{\dagger}(\boldsymbol{\pi} \boldsymbol{\pi}),
$$

which induces

$$
\begin{aligned}
& C_{1}^{\prime \prime} \rightarrow C_{1}^{\prime \prime \prime}=C_{1}^{\prime \prime}-A_{1}^{\prime} \delta_{1}, \\
& C_{2}^{\prime \prime} \rightarrow C_{2}^{\prime \prime \prime}=C_{2}^{\prime \prime}-\left(A_{2}^{\prime}+A_{2}^{\prime *}\right) \delta_{1} .
\end{aligned}
$$

The remaining time derivatives in $D$ and in the $A_{3}$ and $A_{4}$ terms can only be removed if we give up the explicit realization of Lorentz symmetry which we have kept so far. Notice that the time derivatives in the $A_{4}$ term are higher order and can be dropped. The following field redefinition gets rid of the higher order time derivatives in the bilinear terms:

$$
\boldsymbol{\pi}^{i} \mapsto\left(\left(1-\frac{i \partial_{0}}{4 m}+\frac{\boldsymbol{\nabla}^{2}}{8 m^{2}}\right) \delta^{i j}+\frac{\delta_{1} \mathbf{Q}^{i} \mathbf{Q}^{j}}{4 m}\right) \boldsymbol{\pi}^{j}
$$

Finally the time derivatives induced by this redefinition in the four pion terms together with the remaining time derivatives in $A_{3}$ and $A_{4}$ can be removed by

$$
\boldsymbol{\pi} \mapsto \boldsymbol{\pi}+\left(\frac{B_{1}}{2 m}-A_{5}\right) \boldsymbol{\pi}\left(\boldsymbol{\pi}^{\dagger} \boldsymbol{\pi}\right)+\left(\frac{B_{2}}{2 m}-A_{3}\right) \boldsymbol{\pi}^{\dagger}(\boldsymbol{\pi} \boldsymbol{\pi}) .
$$


Putting it all together, we obtain for the constants in the Lagrangian (2.6) the following expressions in terms of the original constants:

$$
\begin{aligned}
\Delta m= & \delta_{1} \mathbf{Q}^{2}\left(1+\left(\delta_{1} A_{0}-\delta_{2}+\frac{\delta_{1}}{2 m}\right) \mathbf{Q}^{2}\right), \\
D_{1}= & \frac{B_{1}}{m}-A_{5}+2 m A_{4}, \\
D_{2}= & \frac{B_{2}}{m}-A_{3}, \\
C_{1}^{\prime}= & C_{1}-B_{2} \delta_{2}+\left(\frac{B_{2}}{m}-A_{3}-A_{1}+2 A_{0} B_{2}\right) \delta_{1}, \\
C_{2}^{\prime}= & C_{2}-2 B_{1} \delta_{2}+\left(\frac{2 B_{1}}{m}-A_{2}-A_{2}^{*}+4 A_{0} B_{1}\right. \\
& \left.-2 A_{5}\right) \delta_{1} .
\end{aligned}
$$

Upon restricting the Lagrangian (2.6) to the zero charge sector we obtain the Lagrangian (2.7) the constants of which are related to the above ones according to

$$
\begin{aligned}
& R_{00}=B_{1}+B_{2}+e^{2}\left(C_{1}^{\prime}+C_{1}^{\prime *}\right)+e^{2} C_{2}^{\prime}, \\
& R_{0 c}=2 B_{2}+2 e^{2} C_{1}^{\prime *}, \\
& R_{c c}=2 B_{1}+4 B_{2}+2 e^{2} C_{3}, \\
& S_{00}=\frac{D_{1}}{2 m}+\frac{D_{2}}{2 m}, \\
& S_{0 c}=\frac{D_{2}}{2 m}, \\
& S_{c c}=\frac{D_{1}}{2 m}+\frac{D_{2}}{m},
\end{aligned}
$$

FIG. 4. Logarithmically divergent diagram which is calculated with the two longitudinal photon propagators (C5) for the dashed lines.

$$
\begin{aligned}
& P_{00}=2 A_{4}, \\
& P_{c c}=2 A_{4} .
\end{aligned}
$$

\section{APPENDIX C: THE COULOMB PROPAGATOR IN $D$ SPACE DIMENSIONS}

We present here a generalization of the Coulomb propagator to $D$ space dimension which may prove useful in bound state calculations. For the actual Coulomb potential in $D$ dimensions

$$
V_{c}(r)=-\frac{\alpha c_{D}}{r^{D-2}} ; \quad c_{D}=\frac{4 \pi \Gamma\left(\frac{D}{2}\right)}{(D-2) 2 \pi^{D / 2}}
$$

we have not been able to find an explicit representation. However, a slight modification of it

$$
V_{c}(r) \rightarrow V_{c}^{\prime}(r)=-\frac{\alpha c_{D}^{\prime}}{r} ; \quad c_{D}^{\prime}=\frac{4 \pi}{\Gamma\left(\frac{D-1}{2}\right)(4 \pi)^{(D-1) / 2}}
$$

admits the following exact representation, which is a generalization of that presented in [27]:

$$
\begin{gathered}
G_{c}(\mathbf{x}, \mathbf{y}, E)=\sum_{l=0}^{\infty} G_{l}(x, y, E) \sum_{\left\{m_{i}\right\}} Y_{l}^{\left\{m_{i}\right\}}\left(\frac{\mathbf{x}}{x}\right) Y_{l}^{*}\left\{m_{i}\right\}\left(\frac{\mathbf{y}}{y}\right), \\
G_{l}(x, y, E)=-m(2 k)^{D-2}(2 k x)^{l}(2 k y)^{l} e^{-k(x+y)} \sum_{s=0}^{\infty} \frac{L_{s}^{2 l+D-2}(2 k x) L_{s}^{2 l+D-2}(2 k y) \Gamma(s+1)}{\left(s+\frac{2 l+D-1}{2}-\frac{m \alpha}{2 k} c_{D}^{\prime}\right) \Gamma(s+2 l+D-1)},
\end{gathered}
$$

where $Y_{l}^{\left\{m_{i}\right\}}$ are the spherical harmonics in $D$ dimensions and $E=-k^{2} / m$. The potential $V_{c}^{\prime}(r)$ corresponds to the following modification of the longitudinal photon propagator in standard DR:

$$
\frac{1}{\mathbf{k}^{2}} \rightarrow\left(\frac{1}{\mathbf{k}^{2}}\right)^{(D-1) / 2}
$$


The change of regularization scheme necessary for translating the result of Eq. (C3) to those of standard DR can be obtained by calculating the logarithmically divergent diagram of Fig. 4 with the two propagators in Eq. (C5). Using the minimal subtraction (MS) renormalization scheme for both regularizations we obtain

$$
\log \frac{\mu^{\prime}}{\mu}=\frac{\gamma_{E}-1-\log (4 \pi)}{2} .
$$

The calculation of $G_{c}(0,0 ; E)$ can be easily done using the formula 1.4.(1) of Ref. [28]

$$
\sum_{n=-\infty}^{\infty} \frac{\Gamma(a+n) \Gamma(b+n)}{\Gamma(c+n) \Gamma(d+n)}=\frac{\pi^{2} \Gamma(c+d-a-b-1)}{\sin (\pi a) \sin (\pi b) \Gamma(c-a) \Gamma(d-a) \Gamma(c-b) \Gamma(d-b)} .
$$

We obtain $\left(D=3+2 \epsilon^{\prime}\right)$

$$
\begin{aligned}
&\left(\mu^{\prime}\right)^{-2 \epsilon^{\prime}} G_{c}\left(\mathbf{0 , 0}-\frac{k^{2}}{m}\right)=-2 m k \frac{2 \pi^{D / 2}}{\Gamma\left(\frac{D}{2}\right)}\left(\frac{2 k}{\mu^{\prime}}\right)^{2 \epsilon^{\prime}} \sum_{s=0}^{\infty} \frac{\Gamma(s+D-1) \Gamma\left(s+\frac{D-1}{2}-\frac{m \alpha}{2 k} c_{D}^{\prime}\right)}{\Gamma(s+1) \Gamma\left(s+\frac{D+1}{2}-\frac{m \alpha}{2 k} c_{D}^{\prime}\right) \Gamma^{2}(D-1)} \\
&=\frac{m k}{4 \pi}\left(\begin{array}{r}
1 \\
+
\end{array}\right. \\
&+\frac{m \alpha}{k}\left[\frac{1}{\epsilon^{\prime}}+2 \log \left(\frac{2 k}{\mu^{\prime}}\right)+2 \gamma_{E}-2 \log (4 \pi)-2\right] \\
& {\left.\left.\left[1+\frac{m \alpha}{2 k}\right)-\psi(1)+\frac{\cos \left(\frac{m \alpha \pi}{2 k}\right)}{\sin \left(\frac{m \alpha \pi}{2 k}\right)}-\frac{2 k}{m \alpha}\right]\right) }
\end{aligned}
$$

Equations (C9), (C10) and (C11) correspond to zero, one and more than one longitudinal photon exchange, respectively. For $E \rightarrow E_{n}=m \alpha^{2} / 4 n^{2}$ we have

$$
\begin{aligned}
& \lim _{E \rightarrow E_{n}}\left(\left(\mu^{\prime}\right)^{-2 \epsilon^{\prime}} G_{c}\left(\mathbf{0 , 0},-\frac{k^{2}}{m}\right)-\frac{\Psi_{n}(\mathbf{0}) \Psi_{n}^{*}(\mathbf{0})}{E-E_{n}}\right)=\frac{m^{2} \alpha}{8 \pi}\left(\frac{1}{n}+\left[2 \log \left(\frac{m \alpha}{n \mu}\right)+\gamma_{E}-\log (4 \pi)-1\right]+\left(2 \psi(n)+2 \gamma_{E}-\frac{3}{n}\right)\right) \\
& :=\frac{m^{2} \alpha \Delta_{n}}{4 \pi}
\end{aligned}
$$

where we have used the MS renormalization scheme and changed $\mu^{\prime}$ by $\mu$ according to Eq. (C6) so that the results above are in standard DR with MS scheme. Clearly the singular part is local, independent of the principal quantum number $n$, and can be absorbed in a renormalization of $R_{c c}^{\prime}$. This result is in agreement with a recent DR calculation of the same object carried out in [29]. Finally formula (5.5) is obtained.

[1] S. Deser et al., Phys. Rev. 96, 774 (1954); T. L. Trueman, Nucl. Phys. 26, 57 (1961); E. Lambert, Helv. Phys. Acta 42, 667 (1969); H. Pilkuhn and S. Wycech, Phys. Lett. 76B, 29 (1978); G. Rasche and W. S. Woolcock, Nucl. Phys. A381, 405 (1982).

[2] A. Gashi, G. Rasche, G. C. Oades, and W. S. Woolcock, Nucl. Phys. A628, 101 (1998); G. Rasche and A. Gashi, Phys. Lett. B 404, 375 (1997).

[3] B. Adeva et al., CERN-SPSLC-95-1; J. Schacher, hep-ph/9808407.
[4] L. L. Nemenov, Yad. Fiz. 41, 980 (1985) [Sov. J. Nucl. Phys. 41, 629 (1985)].

[5] J. Gasser and H. Leutwyler, Ann. Phys. (N.Y.) 158, 142 (1984).

[6] W. E. Caswell and G. P. Lepage, Phys. Lett. 167B, 437 (1986).

[7] L. Afanasyev et al., Phys. Lett. B 308, 200 (1993); 338, 478 (1994).

[8] H. Jallouli and H. Sazdjian, Phys. Rev. D 58, 014011 (1998); 58, 099901(E) (1998). 
[9] M. A. Ivanov, V. E. Lyubovitskij, E. Z. Lipartia, and A. G. Rusetsky, Phys. Rev. D 58, 094024 (1998).

[10] T. Kinoshita and M. Nio, Phys. Rev. D 53, 4909 (1996); P. Labelle, S. M. Zebarjad, and C. P. Burgess, ibid. 56, 8053 (1997); A. H. Hoang, P. Labelle, and S. M. Zebarjad, Phys. Rev. Lett. 79, 3387 (1997).

[11] A. V. Manohar, Phys. Rev. D 56, 230 (1997); A. Pineda and J. Soto, ibid. 58, 114011 (1998).

[12] M. Luke and A. V. Manohar, Phys. Lett. B 286, 348 (1992).

[13] H. B. O'Connell, K. Maltman, A. W. Thomas, and A. G. Williams, hep-ph/9707404.

[14] J. Bijnens, G. Colangelo, G. Ecker, J. Gasser, and M. E. Sainio, Nucl. Phys. B508, 263 (1997); B517, 639(E) (1998); Phys. Lett. B 374, 210 (1996).

[15] M. Knecht and R. Urech, Nucl. Phys. B519, 329 (1998).

[16] X. Kong and F. Ravndal, Phys. Rev. D 59, 014031 (1999).

[17] B. R. Holstein, Phys. Rev. D 60, 114030 (1999).

[18] A. Pineda and J. Soto, Phys. Lett. B 420, 391 (1998); Phys. Rev. D 59, 016005 (1999).

[19] P. Labelle and K. Buckley, hep-ph/9804201.
[20] A. Gall, J. Gasser, V. E. Lyubovitskij, and A. Rusetsky, Phys. Lett. B 462, 335 (1999).

[21] X. Kong and F. Ravndal, Phys. Rev. D 61, 077506 (2000).

[22] Ch. Balzereit, hep-ph/9809226; W. Kilian and T. Ohl, Phys. Rev. D 50, 4649 (1994); C. Balzereit and T. Ohl, Phys. Lett. B 386, 335 (1996).

[23] R. Sundrum, Phys. Rev. D 57, 331 (1998); M. Finkemeier, H. Georgi, and M. McIrvin, ibid. 55, 6933 (1997); C. L. Lee, hep-ph/9709238.

[24] H. Georgi, Nucl. Phys. B361, 339 (1991).

[25] S. Scherer and H. W. Fearing, Phys. Rev. D 52, 6445 (1995).

[26] G. Ecker and M. Mojzis, Phys. Lett. B 410, 266 (1997); 438, 446 (1998).

[27] M. B. Voloshin, Yad. Fiz. 36, 247 (1982) [Sov. J. Nucl. Phys. 36, 143 (1982)]; A. Pineda, Nucl. Phys. B494, 213 (1997).

[28] H. Bateman, Higher Transcendental Functions (McGraw-Hill, New York, 1953), Vol. I.

[29] A. Czarnecki, K. Melnikov, and A. Yelkhovsky, Phys. Rev. A 59, 4316 (1999). 